\title{
Images of a Bose-Einstein condensate in position and momentum space *
}

\author{
Jacek Dziarmaga and Krzysztof Sacha \\ Institute of Physics and Centre for Complex Systems, \\ Jagiellonian University, Reymonta 4, 30-059 Kraków, Poland
}

(Dated: December 08, 2005)

\begin{abstract}
In the Bogoliubov theory a condensate initially prepared in its ground state described by stationary Bogoliubov vacuum and later perturbed by a time-dependent potential or interaction strength evolves into a time-dependent excited state which is dynamical Bogoliubov vacuum. The dynamical vacuum has a simple diagonal form in a time-dependent orthonormal basis of single particle modes. This diagonal representation leads to a gaussian probability distribution for possible outcomes of density measurements in position and momentum space. In these notes we also discuss relations with the $U(1)$ symmetry breaking version of the Bogoliubov theory and give two equivalent gaussian integral representations of the dynamical vacuum state.
\end{abstract}

\section{INTRODUCTION}

It has been established in a number of papers [1, 2] that a density measurement on a Bose-condensed state effectively "collapses" the state to a single Bose-Einstein condensate. The Bose-condensed state before the measurement is a quantum superposition over ideal condensates $\int \mathcal{D} \phi \psi(\phi)|N: \phi\rangle$ with different condensate wave functions $\phi(\vec{x})$ but as position. 1415) s of more and more particles in the state are measured the state of remaining particles gradually collapses towards one of the ideal condensates $|N: \phi\rangle$. As a result the density measurement outcome is $\rho(\vec{x} \mid \phi)=N|\phi(\vec{x})|^{2}$ with probability $P(\phi) \approx|\psi(\phi)|^{2}$.

In our recent paper [3] we developed a measurement theory predicting probability of different density measurement outcomes on a Bose-Einstein condensate evolving under external time-dependent perturbation. At zero temperature in the framework of the Bogoliubov theory the state of the condensate is described by a timedependent dynamical Bogoliubov vacuum. The dynamical vacuum has simple diagonal form which leads to a simple gaussian probability distributions for different density measurement outcomes. The measurement theory for dynamical Bogoliubov vacuum is important because in many experiments, like phase imprinting of dark solitons [4] or condensate splitting in atom interferometers [5], manipulation of the condensate generates substantial dynamical depletion which can qualitatively affect measured density patterns.

\section{DIAGONAL BOGOLIUBOV VACUUM}

According to Bogoliubov theory with well-defined number of atoms [6] a condensate which was initially

\footnotetext{
${ }^{*}$ Talk given at the Laser Physics Workshop, July 2005, Kyoto, Japan
}

prepared in its ground state (i.e. a stationary Bogoliubov vacuum) evolves into a time-dependent excited state which is formally a time-dependent or dynamical Bogoliubov vacuum annihilated by time-dependent Bogoliubov quasiparticle annihilation operators. The dynamical Bogoliubov vacuum can be brought to the diagonal form [3]

$$
\left|0_{b}\right\rangle \sim\left(\hat{a}_{0}^{\dagger} \hat{a}_{0}^{\dagger}+\sum_{\alpha=1}^{M} \lambda_{\alpha} \hat{a}_{\alpha}^{\dagger} \hat{a}_{\alpha}^{\dagger}\right)^{N / 2}|0\rangle,
$$

with a time-dependent orthonormal basis of single particle states $\phi_{\alpha}(t, \vec{x})$ and real time-dependent eigenvalues $\lambda_{\alpha}(t) \in[0,1)$. In the diagonal state (1) we sum over finite number $M$ of non-condensate modes, keeping in mind that $M \rightarrow \infty$. However, in practical calculations which almost always involve some numerics one is forced to work with a finite number of modes. This is why in the following we will keep finite $M$ in all summations without any further comment. As the diagonal vacuum was derived elsewhere [3], here we only mention that $\phi_{0}(t, \vec{x})$ is a condensate wave function solving time-dependent GrossPitaevskii equation and $\phi_{\alpha}(t, \vec{x})$ 's are eigenmodes of the density matrix

$$
\begin{aligned}
& \sum_{m=1}^{M} u_{m}^{*}(t, \vec{x}) u_{m}(t, \vec{y})= \\
& \sum_{\alpha=1}^{M}\left[1+\mathrm{d} N_{\alpha}(t)\right] \phi_{\alpha}^{*}(t, \vec{x}) \phi_{\alpha}(t, \vec{y}) .
\end{aligned}
$$

Here $\left[u_{m}(t, \vec{x}), v_{m}(t, \vec{x})\right]$ 's are Bogoliubov modes solving time-dependent Bogoliubov-de Gennes equations. In the number-conserving Bogoliubov theory modes $u_{m}(t, \vec{x})$ and $v_{m}^{*}(t, \vec{x})$ are orthogonal to the condensate wave function $\phi_{0}(t, \vec{x}) . \mathrm{d} N_{\alpha}(t)$ is average number of atoms depleted from the condensate wave function $\phi_{0}(t, \vec{x})$ to the mode $\phi_{\alpha}(t, \vec{x})$. The eigenvalues $\lambda_{\alpha}(t)$ in the diagonal vacuum (1) are

$$
\lambda_{\alpha}=\sqrt{\frac{\mathrm{d} N_{\alpha}}{1+\mathrm{d} N_{\alpha}}} .
$$


Phases of the modes $\phi_{\alpha}(t, \vec{x})$ are chosen in such a way that the eigenvalues of the matrix

$$
\begin{aligned}
& \sum_{m=1}^{M} v_{m}^{*}(t, \vec{x}) u_{m}(t, \vec{y})= \\
& \sum_{\alpha=1}^{M} \sqrt{\mathrm{d} N_{\alpha}(t)\left[1+\mathrm{d} N_{\alpha}(t)\right]} \phi_{\alpha}(t, \vec{x}) \phi_{\alpha}(t, \vec{y}) .
\end{aligned}
$$

are real and positive. For the sake of completeness we also quote here the diagonal density matrix for noncondensate modes

$$
\begin{aligned}
& \sum_{m=1}^{M} v_{m}(t, \vec{x}) v_{m}^{*}(t, \vec{y})= \\
& \sum_{\alpha=1}^{M} \mathrm{~d} N_{\alpha}(t) \phi_{\alpha}^{*}(t, \vec{x}) \phi_{\alpha}(t, \vec{y}) .
\end{aligned}
$$

One of the results of Ref. 3 ] is that the operators (245) can be simultaneously diagonalized by the same single particle non-condensate modes $\phi_{\alpha}(t, \vec{x})$. Phases of these modes are uniquely defined by the requirement that the eigenvalues of the operator (4) are real and positive.

\section{SUPERPOSITION OVER CONDENSATES}

Having in mind applications in quantum measurement theory it is useful to rewrite the diagonal state (1D) as a gaussian superposition over $N$-particle condensates

$$
\left|0_{b}\right\rangle \sim \int d q e^{-\sum_{\alpha=1}^{M} \frac{1-\lambda_{\alpha}}{2 \lambda_{\alpha}} q_{\alpha}^{2}}|N: \phi(\vec{x} \mid q)\rangle .
$$

with the normalized condensate wave functions

$$
\phi(\vec{x} \mid q)=\frac{\phi_{0}(\vec{x})+\frac{1}{\sqrt{N}} \sum_{\alpha=1}^{M} q_{\alpha} \phi_{\alpha}(\vec{x})}{\sqrt{1+\frac{1}{N} \sum_{\beta=1}^{M} q_{\beta}^{2}}} .
$$

Here the state $|N: \phi(\vec{x} \mid q)\rangle$ is perfect condensate with all $N$ particles in the same condensate wave function $\phi(\vec{x} \mid q)$. The (rather technical) proof of the equivalence between the diagonal vacuum in Eq.(11) and the gaussian superposition in Eq.(7) is given in Ref. [3]. The equivalence is approximate, it requires that the average number of depleted particles is much less than the total number of particles,

$$
\mathrm{d} N \equiv \sum_{\alpha=1}^{M} \mathrm{~d} N_{\alpha} \ll N .
$$

This is not a new assumption but the usual requirement in the Bogoliubov theory.

\section{CONNECTION WITH THE SYMMETRY BREAKING APPROACH}

This section is a brief digression on the standard $U(1)$ symmetry breaking version of the Bogoliubov theory where one splits the field operator into a $c$-number condensate part plus a small quantum fluctuation

$$
\hat{\Psi}(\vec{x})=\sqrt{N} \phi_{0}(\vec{x})+\hat{\psi}(\vec{x}) .
$$

The quantum fluctuation is further Bogoliubov transformed as

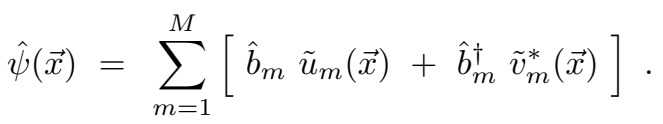

The initial stationary Bogoliubov vacuum state evolves into a time-dependent Bogoliubov vacuum state annihilated by the operators $b_{m}$. In the $U(1)$ symmetry breaking Bogoliubov theory the reduced single particle density matrix in the time-dependent vacuum state is

$$
\left\langle\hat{\psi}^{\dagger}(\vec{x}) \hat{\psi}(\vec{y})\right\rangle=\sum_{m=1}^{M} \tilde{v}_{m}(t, \vec{x}) \tilde{v}_{m}^{*}(t, \vec{y})
$$

and the anomalous density matrix is

$$
\langle\hat{\psi}(\vec{x}) \hat{\psi}(\vec{y})\rangle=\sum_{m=1}^{M} \tilde{u}_{m}(t, \vec{x}) \tilde{v}_{m}^{*}(t, \vec{y}) .
$$

However, these density matrices are not confined to the subspace orthogonal to the condensate wave function $\phi_{0}(t, \vec{x})$ because, unlike in the number-conserving theory, here the Bogoliubov modes $\tilde{u}_{m}(t, \vec{x})$ and $v_{m}^{*}(t, \vec{x})$ are not exactly orthogonal to $\phi_{0}(t, \vec{x})$. This error can be corrected using the relations

$$
\begin{aligned}
& u_{m}(\vec{x})=\tilde{u}_{m}(\vec{x})-\phi_{0}(\vec{x})\left\langle\phi_{0} \mid \tilde{u}_{m}\right\rangle \equiv Q_{\vec{x}} u_{m}(\vec{x}), \\
& v_{m}(\vec{x})=\tilde{v}_{m}(\vec{x})-\phi_{0}^{*}(\vec{x})\left\langle\phi_{0}^{*} \mid \tilde{v}_{m}\right\rangle \equiv Q_{\vec{x}}^{*} v_{m}(\vec{x})
\end{aligned}
$$

between Bogoliubov modes in the number-conserving and the symmetry-breaking theories [6]. After the projection on the subspace orthogonal to $\phi_{0}(t, \vec{x})$ we obtain the correct non-condensate density matrix

$$
\begin{aligned}
& Q_{\vec{x}}^{*} Q_{\vec{y}}\left\langle\hat{\psi}^{\dagger}(\vec{x}) \hat{\psi}(\vec{y})\right\rangle= \\
& \sum_{m=1}^{\infty} v_{m}(t, \vec{x}) v_{m}^{*}(t, \vec{y})= \\
& \sum_{\alpha=1}^{\infty} \mathrm{d} N_{\alpha}(t) \phi_{\alpha}^{*}(t, \vec{x}) \phi_{\alpha}(t, \vec{y})
\end{aligned}
$$

and the correct anomalous density matrix

$$
\begin{aligned}
& Q_{\vec{x}} Q_{\vec{y}}\langle\hat{\psi}(\vec{x}) \hat{\psi}(\vec{y})\rangle= \\
& \sum_{m=1}^{\infty} u_{m}(t, \vec{x}) v_{m}^{*}(t, \vec{y})= \\
& \sum_{\alpha=1}^{\infty} \sqrt{\mathrm{d} N_{\alpha}(t)\left[1+\mathrm{d} N_{\alpha}(t)\right]} \phi_{\alpha}(t, \vec{x}) \phi_{\alpha}(t, \vec{y}) .
\end{aligned}
$$


The last equalities in Eqs.1415) follow from equations (54) in the number-conserving theory.

Results of the conventional $U(1)$ symmetry breaking theory can be translated into correct results of the number-conserving theory either by

- projecting the Bogoliubov modes on the subspace orthogonal to the condensate wave function like in Eqs.(13), or

- projecting directly the density matrices $\left\langle\hat{\psi}^{\dagger}(\vec{x}) \hat{\psi}(\vec{y})\right\rangle$ and $\langle\hat{\psi}(\vec{x}) \hat{\psi}(\vec{y})\rangle$ in Eqs. (1112) in a way described by Eqs.1415,

or any other way to elliminate contamination by the condensate wave function $\phi_{0}(t, \vec{x})$ of what is wrongly believed to be purely non-condensate density matrices.

\section{BOGOLIUBOV REPRESENTATION}

We have shown that in the limit of large $N$ the two forms (11) and (6) of the Bogoliubov vacuum are equivalent. However, there is yet another representation of the vacuum which may appeal more to some of the readers because it is very similar to the coherent state representation of the vacuum in quantum optics. The representation is 7$]$

$$
\left|0_{b}\right\rangle \sim \int d^{2} b e^{-\frac{1}{2} b^{*} b}|N: \phi(\vec{x} \mid b)\rangle .
$$

Here $d^{2} b=\prod_{m=1}^{M} d^{2} b_{m}, b^{*} b=\sum_{m=1}^{M} b_{m}^{*} b_{m}$ and $\phi(\vec{x} \mid b)$ is a normalized condensate wave function

$$
\begin{aligned}
& \phi(\vec{x} \mid b)= \\
& \mathcal{N}\left\{\phi_{0}(t, \vec{x})+\frac{1}{\sqrt{N}} \sum_{m=1}^{M}\left[b_{m} u_{m}(t, \vec{x})+b_{m}^{*} v_{m}^{*}(t, \vec{x})\right]\right\}
\end{aligned}
$$

where the normalization factor is

$$
\mathcal{N}^{-1}=1+\frac{1}{N} \sum_{m, n=1}^{M}\left\langle b_{m} u_{m}+b_{m}^{*} v_{m}^{*} \mid b_{n} u_{n}+b_{n}^{*} v_{n}^{*}\right\rangle \text {. }
$$

In this Section we will prove equivalence between Eq. (16) and Eq. (6) in the limit of large $N$. The main difference between these equations is that in Eq.(6) there are $M$ integrations over real coordinates $q_{\alpha}$ while in Eq.(16) there are also $M$ integrations but over complex coordinates $b_{m}$. In this sense Eq. (6) is more compact representation than Eq.(16).

As a first step we use the large $N$ limit to transfer the normalization factor $\mathcal{N}$ to the exponent in Eq.(16),

$$
\begin{array}{r}
\int d^{2} b \quad e^{-\frac{1}{2} \sum_{m} b_{m}^{*} b_{m}-\frac{1}{2} \sum_{m n}\left\langle b_{m} u_{m}+b_{m}^{*} v_{m}^{*} \mid b_{n} u_{n}+b_{n}^{*} v_{n}^{*}\right\rangle} \\
\left(\hat{a}_{0}^{\dagger}+\frac{1}{\sqrt{N}} \sum_{m=1}^{M}\left[b_{m} \hat{u}_{m}^{\dagger}+b_{m}^{*} \hat{v}_{m}^{\dagger}\right]\right)^{N}|0\rangle \cdot(19)
\end{array}
$$

Here the new operators are $\hat{u}_{m}=\left\langle u_{m} \mid \hat{\psi}\right\rangle$ and $\hat{v}_{m}=\left\langle v_{m}^{*} \mid \hat{\psi}\right\rangle$. At this point we make a transformation to the orthonormal basis of $\phi_{\alpha}$ 's, $\sum_{m=1}^{M}\left[b_{m} \hat{u}_{m}^{\dagger}+b_{m}^{*} \hat{v}_{m}^{\dagger}=\right] \sum_{\alpha=1}^{M} z_{\alpha} \hat{a}_{\alpha}^{\dagger}$, or equivalently

$$
\sum_{m}\left[b_{m} u_{m}+b_{m}^{*} v_{m}^{*}\right]=\sum_{\alpha} z_{\alpha} \phi_{\alpha} .
$$

After this transformation the state (19) becomes

$$
\begin{aligned}
\int d^{2} z & e^{-\frac{1}{2} \sum_{m} b_{m}^{*} b_{m}-\frac{1}{2} \sum_{\alpha} z_{\alpha}^{*} z_{\alpha}} \\
& \left(\hat{a}_{0}^{\dagger}+\frac{1}{\sqrt{N}} \sum_{\alpha=1}^{M} z_{\alpha} \hat{a}_{\alpha}^{\dagger}\right)^{N}|0\rangle .
\end{aligned}
$$

Here $b$ 's are linear functions of $z$ 's

$$
b_{m}=\sum_{\alpha=1}^{M}\left[z_{\alpha}\left\langle u_{m} \mid \phi_{\alpha}\right\rangle-z_{\alpha}^{*}\left\langle v_{m} \mid \phi_{\alpha}^{*}\right\rangle\right]
$$

obtained by inverting the transformation (20). Using this inverse transformation and equations (245) we rewrite the exponent in Eq.(21) as

$$
\begin{aligned}
& \sum_{m} b_{m}^{*} b_{m}+\sum_{\alpha} z_{\alpha}^{*} z_{\alpha}= \\
& \sum_{\alpha} 2\left(1+\mathrm{d} N_{\alpha}\right) z_{\alpha}^{*} z_{\alpha}- \\
& \sqrt{\mathrm{d} N_{\alpha}\left(1+\mathrm{d} N_{\alpha}\right)} z_{\alpha}^{*} z_{\alpha}^{*}-\sqrt{\mathrm{d} N_{\alpha}\left(1+\mathrm{d} N_{\alpha}\right)} z_{\alpha} z_{\alpha}
\end{aligned}
$$

With this exponent the gaussian integral in Eq.(21) gives a simple correlator for $z$ 's

$$
\begin{gathered}
\int d^{2} z e^{-\frac{1}{2} \sum_{m} b_{m}^{*} b_{m}-\frac{1}{2} \sum_{\alpha} z_{\alpha}^{*} z_{\alpha}} z_{\beta} z_{\gamma}= \\
\delta_{\beta \gamma} \sqrt{\frac{\mathrm{d} N_{\beta}}{1+\mathrm{d} N_{\beta}}}=\delta_{\beta \gamma} \lambda_{\beta} .
\end{gathered}
$$

In the last equality we use the relation in Eq.(3). The gaussian state in Eq.21) is completely determined by this correlator. The same correlator for real $q$ 's is also obtained in the real gaussian integral

$$
\int d q e^{-\frac{1}{2} \sum_{\alpha} \frac{q_{\alpha}^{2}}{\lambda_{\alpha}}} q_{\beta} q_{\gamma}=\delta_{\beta \gamma} \lambda_{\beta} .
$$

Comparing the correlators (24) and (25) we find that the gaussian state (21) is equal to the state

$$
\int d q e^{-\frac{1}{2} \sum_{\alpha} \frac{q_{\alpha}^{2}}{\lambda_{\alpha}}}\left(\hat{a}_{0}^{\dagger}+\frac{1}{\sqrt{N}} \sum_{\alpha=1}^{M} q_{\alpha} \hat{a}_{\alpha}^{\dagger}\right)^{N}|0\rangle
$$

with real coordinates $q$. Finally after normalization of the creation operator we get

$$
\int d q e^{-\sum_{\alpha} \frac{\left(1-\lambda_{\alpha}\right) q_{\alpha}^{2}}{2 \lambda_{\alpha}}}\left(\frac{\hat{a}_{0}^{\dagger}+\frac{1}{\sqrt{N}} \sum_{\alpha=1}^{M} q_{\alpha} \hat{a}_{\alpha}^{\dagger}}{\sqrt{1+\frac{1}{N} \sum_{\beta=1}^{M} q_{\beta}^{2}}}\right)^{N}
$$


and this state is the gaussian representation of Bogoliubov vacuum in Eq.(6). In this way we have shown that the representations (6) and (16) are equivalent.

The complex representation (16) with Bogoliubov coefficients $b_{m}$ has twice as many integration variables as the real representation (6) with real coordinates $q_{\alpha}$. What is more, the real representation (6) is spanned by the orthonormal basis of $\phi_{\alpha}$ 's while the complex representation is spanned by the Bogoliubov modes $\left(u_{m}, v_{m}\right)$ which are not orthogonal in the usual sense. The real representation reveals the diagonal structure of the vacuum which is implicit in the complex representation.

\section{PROBABILITY DISTRIBUTION}

Having shown equivalence between different representations of the dynamical Bogoliubov vacuum (16 16) we now return to the main subject of this paper which is probability distribution for different condensate wave functions. The quantum superposition in the real representation (6) directly leads to the probability distribution for different $q$ 's

$$
P(q) \propto \prod_{\alpha=1}^{M} e^{-\frac{q_{\alpha}^{2}}{2 \mathrm{~d} N_{\alpha}}}
$$

which is valid for the non-condensate modes with large occupation numbers $\mathrm{d} N_{\alpha}$ when we can replace

$$
\frac{1-\lambda_{\alpha}}{2 \lambda_{\alpha}} \approx \frac{1}{4 \mathrm{~d} N_{\alpha}}
$$

compare Eq.(3). The distribution $P(q)$ is the desired probability distribution for different possible density measurement outcomes given by

$$
\begin{aligned}
& \rho(\vec{x} \mid q)=N|\phi(\vec{x} \mid q)|^{2}= \\
& N\left|\frac{\phi_{0}(t, \vec{x})+\frac{1}{\sqrt{N}} \sum_{\alpha=1}^{M} q_{\alpha} \phi_{\alpha}(t, \vec{x})}{\sqrt{1+\frac{1}{N} \sum_{\beta=1}^{N} q_{\beta}^{2}}}\right|^{2} .
\end{aligned}
$$

Equations (28) and (30) define a simple scheme how to simulate possible density measurement outcomes on the dynamical Bogoliubov vacuum state.

\section{MEASUREMENTS IN MOMENTUM SPACE}

Up to this point we assumed ideal density measurement where all particle positions are measured at the same time. However, there is no reason why these results should be limited to measurements of particle momenta instead of position. In fact the diagonal second quantized representation (1) prefers neither position nor momentum representation, and consequently all the following formulas can be rewritten in momentum representation simply by replacing $\vec{x} \rightarrow \vec{k}$ and $\vec{y} \rightarrow \vec{p}$. In the end we arrive at the probability distribution $P(q)$ in Eq. (28) for different possible outcomes of momentum distribution

$$
\rho(\vec{k} \mid q)=N\left|\frac{\phi_{0}(t, \vec{k})+\frac{1}{\sqrt{N}} \sum_{\alpha=1}^{M} q_{\alpha} \phi_{\alpha}(t, \vec{k})}{\sqrt{1+\frac{1}{N} \sum_{\beta=1}^{N} q_{\beta}^{2}}}\right|^{2} .
$$

A quantum measurement on the dynamical Bogoliubov vacuum state which measures all particle momenta at the same time will give momentum density distributions (31) with probabilities (28).

\section{CONCLUSION}

In conclusion, we derived a convenient diagonal form of the time-dependent Bogoliubov vacuum which greatly facilitates simulations of quantum measurements on Bosecondensed systems both in position and momentum space.

Acknowledgements. - Work of JD was supported in part by the KBN grant PBZ-MIN-008/P03/2003 and work of KS was supported in part by Polish Government scientific funds (2005-2008) as a research project.

[1] J. Javanainen and S.M. Yoo, Phys. Rev. Lett. 76, 161 (1996).

[2] C. J. Pethick and H. Smith, Bose-Einstein Condensation in Dilute Gases, (Cambridge Univ. Press, 2002); Y. Castin and J. Dalibard, Phys. Rev. A 55, 4330 (1997); K. Mølmer, Phys. Rev. A 65, 021607 (2002); S. Ashhab and A.J. Leggett, Phys. Rev. A 65, 023604 (2002).

[3] J. Dziarmaga and K. Sacha, J.Phys.B: At.Mol.Opt.Phys. 39, 43 (2006).

[4] S. Burger et al., Phys. Rev. Lett. 83, 5198 (1999).

[5] Y. Shin et al., Phys. Rev. Lett. 92, 050405 (2004).

[6] Y. Castin and R. Dum, Phys. Rev. A 57, 3008 (1998); J. Dziarmaga and K. Sacha, Phys. Rev. A 67033608 (2003).

[7] J. Dziarmaga and J. Meisner, J.Phys.B: At.Mol.Opt.Phys. 38, 4211 (2005). 